\title{
Luchas por el derecho a la salud en Colombia. Vínculos con la salud para todos y todas
}

\author{
Struggles for the right to health in Colombia. Links with health for all \\ Mauricio Torres-Tovar1, Román Rafael Vega-Romero $\mathbf{2}$, Jairo Ernesto Luna-García1, Yadira Eugenia \\ Borrero-Ramírez $\mathbf{3}^{\mathbf{3}}$ María Esperanza Echeverry-López ${ }^{\mathbf{3}}$
}

DOI: $10.1590 / 0103-110420205104$

\begin{abstract}
RESUMEN Se desarrolló una investigación en Colombia cuyo objetivo fue conocer el involucramiento de la sociedad civil en el trabajo por la salud para todos, en las siguientes dimensiones: construcción del movimiento por la salud en espacios locales y nacionales; campañas y abogacía; generación, diseminación y uso de conocimiento; entrenamiento y construcción de capacidades; y diálogo sobre las políticas e involucramiento de la sociedad civil en la gobernanza global. Fue una investigación cualitativa que contó con diferentes enfoques metodológicos y aplicación de métodos. Incluyó siete casos de experiencias del involucramiento de la sociedad civil en el logro de la salud para todos en espacios locales -urbanos y rurales- con comunidades indígenas, campesinas, LGTB, populares y trabajadoras. Los hallazgos permiten concluir que se da un enriquecido y heterogéneo panorama de involucramiento de procesos organizativos y movimientos sociales de la sociedad civil en la lucha por la salud para todos en el ámbito local, regional y nacional. Se identifican modos diversos de acción colectiva, según las condiciones de clase, género, étnicas, laborales y ciudadanas, que muestran variadas formas autóctonas de involucramiento de la sociedad civil, con sus propios elementos de actuación, resistencias y logros.
\end{abstract}

PALABRAS CLAVES Derecho a la salud. Participación de la comunidad. Políticas de salud. Colombia.

1 Universidad Nacional de Colombia- Bogota, Colombia.

mhtorrest@unal.edu.co

2 Universidad Pontificia Javeriana-Bogota,

Colombia.

3 Universidad de Antioquia,

Facultad Nacional de

Salud Pública - Medellín,

Colombia.
ABSTRACT An investigation was carried out in Colombia whose objective was to know the involvement of civil society in the struggle for health for all, in the following dimensions: construction of the movement for health in local and national spaces; campaigns and advocacy; generation, dissemination and use of knowledge; training and capacity building; and policy dialogue and the involvement of civil society in global governance. It was a qualitative investigation that had different methodological approaches and application of methods. It included seven cases of experiences of civil society involvement in achieving health for all in local spaces -urban and rural- with indigenous, peasant, LGTB, popular and working communities. The findings allow us to conclude that there is a rich and heterogeneous panorama of the involvement of organizational processes and social movements of civil society in the fight for health for all at the local, regional and national levels. Different modes of collective action were identified, according to the class, gender, ethnic, labor and citizenship conditions, which show various native forms of civil society involvement, with their own elements of action, resistance and achievements.

KEYSWORDS Right to health. Community participation. Health policy. Colombia. 


\section{Introducción}

La persistencia e incremento de importantes desigualdades en salud entre países y al interior de ellos, motivó el debate frente a la vigencia y necesidad de la Atención Primaria en Salud, formulada en Alma-Ata en 1978 1,2 y la incorporación de la salud en el ámbito de los Objetivos de Desarrollo Sostenible (ODS) en la agenda post $2015^{3}$.

Desde 1980 y especialmente a partir de la Primera Asamblea del Movimiento de Salud de los Pueblos en el año 2000, se configura un proceso encaminado a fortalecer los vínculos entre organizaciones de base del ámbito local, nacional y global, y crear un movimiento global para reposicionar los objetivos de la salud dentro de la agenda de desarrollo internacional ${ }^{4}$. Este proceso ha tenido como eje prioritario el fortalecimiento de la participación de la sociedad civil para lograr la salud para todos ${ }^{5}$.

Esta investigación aborda el caso colombiano, como parte de un proyecto internacional implementado por el Movimiento de Salud de los Pueblos en seis países del mundo. Su objetivo fue explorar, con base en cinco categorías ${ }^{6}$ (que se describen más adelante), cómo el involucramiento de la sociedad civil aporta al logro de la salud para todos ${ }^{7}$.

Colombia, según la Constitución Política de 1991, es un Estado Social de Derecho, organizado como República unitaria, descentralizada, democrática, participativa y pluralista, con un sistema político presidencialista. Esta formulación se confronta con la realidad de una democracia representativa limitada, de tradición bipartidista, excluyente de sectores políticos alternativos, con escasa promoción de la participación ciudadana que deviene en decisiones tomadas por élites orientadas a intereses particulares y patrimonialistas, generando profundas injusticias sociales, base de un duradero conflicto social, político y armado de más de 50 años en el país ${ }^{8}$.
En este contexto, desde finales de la década de 1980, Colombia inició, siguiendo orientaciones de política promovidas por organismos multilaterales de crédito, el Banco Mundial (BM) y el Banco Interamericano de Desarrollo (BID) 9 , un largo proceso de reformas económicas y de recorte de la política social, articuladas por la maximización del mercado, bajo el argumento de promover un ajuste estructural para superar la crisis de la deuda externa. Esto ha generado procesos de resistencia y movilización social por la salud, y la constitución de un movimiento nacional por la salud en Colombia ${ }^{\mathbf{1 0}, 11}$.

En ese escenario, el objetivo de esta investigación fue el estudio de siete casos representativos del involucramiento de la sociedad civil en la lucha por la salud para todos: la Asociación de Trabajadores Enfermos y Extrabajadores de Colmotores (Asotrecol); el proceso de resistencia de los trabajadores del Hospital San Juan de Dios (HSJD); el movimiento de lesbianas, gays, transexuales y bisexuales (LGTB) de Cali; la Escuela Popular de Líderes de Salud (EPLS) adscrita a la Mesa Intersectorial de Antioquia por el Derecho a la Salud (Mias); la Asociación Campesina del Valle del Río Cimitarra (ACVC); la Asociación de Cabildos Indígenas del Norte del Cauca (Acin); y el Movimiento Nacional por la Salud y la Seguridad Social (MNSSS) ${ }^{12-20}$.

Para el estudio de estos casos se abordaron cinco categorías teórico-empíricas definidas en la investigación internacional6: i) construcción del movimiento por la salud en espacios locales y nacionales, entendida como la trayectoria en el contexto mencionado, con sus logros y dificultades; ii) campañas y abogacía, que se refieren al activismo en salud o en asuntos relacionados con los determinantes sociales de la salud, y representa la agenda y la articulación interna y con otros actores; iii) generación, diseminación y uso de conocimiento, que busca comprender la producción, el uso, transferencia, impacto y contribución del 
conocimiento al movimiento; iv) entrenamiento y construcción de capacidades, que aborda los aportes de las iniciativas educativas a la acción social y política; y, v) diálogo sobre las políticas y el involucramiento de la sociedad civil en la gobernanza global, que hace referencia a la incidencia de las organizaciones en la toma de decisiones globales -por ejemplo, de las Organizaciones Mundial y Panamericana de la Salud-, y en el fortalecimiento de la voz de los gobiernos del sur global, cuyo alcance en esta investigación nacional es el de la incidencia de los movimientos y organizaciones sociales estudiados en las políticas nacionales o locales de salud.

Ese involucramiento se ha desplegado, principalmente, a través de contiendas por el derecho a la salud, que evidencian el surgimiento y fortalecimiento de diversos actores colectivos locales y regionales, y uno nacional-Movimiento Nacional por la Salud y la Seguridad Social-, cuya centralidad es la lucha por ese derecho, o la incorporación de la salud a sus agendas de defensa del territorio, de una cosmogonía propia, de la diversidad sexual, y de Determinantes Sociales de la Salud (DSS), como el medio ambiente, el derecho al trabajo, y la soberanía alimentaria.Estos movimientos han usado repertorios disruptivos e institucionales para visibilizar y tramitar esas demandas, construyendo redes de solidaridad y colaboración entre actores a través de diversas tradiciones de lucha,frente a las reformas económicas y sociales regresivas que se han producido en el país.

La polifonía del involucramiento, aborda en este artículo procesos organizativos urbanos, rurales, étnicos, laborales, de educación popular, y de articulación de un actor colectivo nacional, y evidencia los claroscuros de procesos organizativos, de movilización y resistencia construidos en el contexto colombiano, marcado por el despojo de derechos sociales y de violencia socio-política.

\section{Material y métodos}

Se llevó a cabo una investigación cualitativa con distintos enfoques metodológicos y aplicación de métodos, como sistematización de experiencias, etnografía, biografías, evaluación realista, investigación acción, entre otros $^{21}$. El estudio incluyó siete casos de experiencias del involucramiento de la sociedad civil en el logro del derecho a la salud y de la salud para todos, en espacios urbanos y rurales que se refirieron a comunidades indígenas, campesinas, mujeres, LGTB y trabajadores, y un caso del orden nacional. Las técnicas de recolección de información involucraron análisis documental, grupos focales, entrevistas semiestructuradas y observación participante.

En la tarea de construir un marco conceptual de análisis, si bien se partió del propuesto por el proyecto global del Movimiento de Salud de los Pueblos, cada investigación tuvo referentes teóricos y enfoques particulares que involucraron, de acuerdo al caso, teorías sobre la Acción Colectiva (AC), la resistencia, la subalteridad, la construcción social de la realidad, la determinación social de la salud, el derecho a la salud, la salud de los trabajadores y el género. A su vez, el equipo de investigación construyó documentos guía relacionados con i) orientaciones para la construcción del informe de cada uno de los estudios de caso; ii) análisis integrado de la información de los casos de Colombia; iii) comprensión de la noción de sociedad civil; iv) categorías de análisis adoptadas del proyecto del Movimiento de Salud de los Pueblos sobre involucramiento de la sociedad civil en el logro de la salud para todos ya revisadas en la introducción; y, v) propuesta de estructura para el documento integrado del caso Colombia.

Es importante mencionar que el estudio se desarrolló en el contexto de la investigación internacional del Movimiento deSalud de los Pueblos, el cual incluyó una evaluación empírica de los aspectos organizativos, 
elementos de práctica, estrategias y apoyos financieros del involucramiento de la sociedad civil en el logro de la salud para todos a través de sus cinco programas -apoyo a los círculos de país, universidad internacional de la salud de los pueblos, campaña por la salud para todos, democratización de la gobernanza en salud y observatorio global de salud-, con énfasis en los casos de la India, Italia, Sudáfrica, Congo, Brasil y Colombia.

\section{Resultados y discusión}

A continuación se presentan los resultados de los siete casos de involucramiento de la sociedad civil en la salud para todos estudiados en Colombia, los cuales se organizan de acuerdo a las cinco categorías teórico-empíricas presentadas en la introducción.

\section{Construcción de movimiento por la salud en espacios nacionales y locales}

En el marco de la contienda por el derecho a la salud y por alcanzar la salud para todos, se han configurado en Colombia múltiples actores colectivos, algunos del ámbito nacional, pero la mayoría de ellos corresponden a procesos organizativos locales -tanto urbanos como rurales-, los cuales se caracterizan por recoger diferentes tradiciones de lucha por el derecho a la salud y a la seguridad social, al territorio, a la vida, a la soberanía alimentaria, a la salud pública, a las cosmovisiones y prácticas ancestrales, entre otros.

Estos temas han devenido parte de la agenda de lucha de las organizaciones y movimientos sociales, dados los problemas de acceso y reconocimiento de derechos,en el marco de la reforma sanitaria de la década de 1990 y de la violencia propia del conflicto armado interno. Como parte de estos procesos, los movimientos y organizaciones han construido la noción de la salud como derecho fundamental e interdependiente; han utilizado repertorios de lucha tanto contenciosos (marchas, plantones, toma de vías o instituciones, huelgas, etc.) como no contenciosos (resolución por canales institucionales, como la tutela ${ }^{22}$, entre otros); y sus actores se han organizado y tejido lazos de solidaridad y trabajo colaborativo para desarrollar la lucha. Cada uno de estos procesos organizativos tiene particularidades que se presentan en la cuadro 1.

Cuadro 1. Particularidades organizativas y de lucha por la salud en Colombia

\begin{tabular}{|c|c|c|c|c|c|}
\hline $\begin{array}{l}\text { Tipo de actores que lo } \\
\text { conforman }\end{array}$ & $\begin{array}{l}\text { Tradiciones de lucha } \\
\text { que recoge }\end{array}$ & $\begin{array}{l}\text { Periodo de origen } \\
\text { del proceso }\end{array}$ & $\begin{array}{l}\text { Principales razones para } \\
\text { luchar por la salud }\end{array}$ & $\begin{array}{l}\text { Prioridades de las } \\
\text { demandas en salud }\end{array}$ & $\begin{array}{l}\text { Ámbito de } \\
\text { acción }\end{array}$ \\
\hline \multicolumn{6}{|c|}{ Movimiento Nacional por la Salud y la Seguridad Social } \\
\hline $\begin{array}{l}\text { Organizaciones sin- } \\
\text { dicales nacionales y } \\
\text { locales, campesinas, } \\
\text { indígenas, afro, de } \\
\text { mujeres, de profesiona- } \\
\text { les y trabajadores de la } \\
\text { salud, estudiantiles, de } \\
\text { usuarios de servicios de } \\
\text { salud y de pacientes de } \\
\text { alto costo, y movimien- } \\
\text { to por la paz. }\end{array}$ & $\begin{array}{l}\text { Luchas sindicales desde } \\
\text { la década de 1970, or- } \\
\text { ganizaciones de partici- } \\
\text { pación comunitaria en } \\
\text { salud, organizaciones } \\
\text { no gubernamentales, } \\
\text { luchas campesinas } \\
\text { e indígenas desde la } \\
\text { década de 1960, organi- } \\
\text { zaciones de salubristas, } \\
\text { agremiaciones profesio- } \\
\text { nales de la salud. }\end{array}$ & $\begin{array}{l}\text { Desde finales de } \\
\text { la década de } 1990, \\
\text { con periodos de } \\
\text { invisibilidad y otros } \\
\text { de mayor visibi- } \\
\text { lidad en la esfera } \\
\text { pública. }\end{array}$ & $\begin{array}{l}\text { Deterioro de los indicado- } \\
\text { res de salud pública. } \\
\text { Mercantilización de los } \\
\text { servicios de salud. } \\
\text { Barreras de acceso a la } \\
\text { atención. } \\
\text { Reproducción en el sistema } \\
\text { de salud de las inequidades } \\
\text { sociales. } \\
\text { Defensa del territorio y de } \\
\text { la vida }\end{array}$ & $\begin{array}{l}\text { Salud como derecho } \\
\text { fundamental, sin restric- } \\
\text { ciones en el acceso. } \\
\text { Mejorar los determinan- } \\
\text { tes de la salud. } \\
\text { Defensa de las institucio- } \\
\text { nes públicas de salud. } \\
\text { Equidad en salud desde } \\
\text { una perspectiva de justi- } \\
\text { cia distributiva. }\end{array}$ & $\begin{array}{l}\text { De carácter } \\
\text { nacional, con } \\
\text { presencia es- } \\
\text { pecialmente en } \\
\text { las principales } \\
\text { capitales. }\end{array}$ \\
\hline
\end{tabular}


Cuadro 1. (cont.)

\begin{tabular}{|c|c|c|c|}
\hline $\begin{array}{l}\text { Tipo de actores que lo } \\
\text { conforman }\end{array}$ & $\begin{array}{l}\text { Tradiciones de lucha } \\
\text { que recoge }\end{array}$ & $\begin{array}{l}\text { Periodo de origen } \\
\text { del proceso }\end{array}$ & $\begin{array}{l}\text { Principales razones para } \\
\text { luchar por la salud }\end{array}$ \\
\hline \multicolumn{4}{|c|}{ Hospital San Juan de Dios (HSJD) } \\
\hline $\begin{array}{l}\text { Trabajadores del hos- } \\
\text { pital, especialmente au- } \\
\text { xiliares de enfermería. }\end{array}$ & $\begin{array}{l}\text { Luchas de los trabaja- } \\
\text { dores y estudiantes del } \\
\text { HSJD. }\end{array}$ & $\begin{array}{l}\text { Finales de la déca- } \\
\text { da de } 1990 \text { por la } \\
\text { crisis hospitalaria } \\
\text { nacional, pero se } \\
\text { consolida a partir } \\
\text { del cierre del HSJD } \\
\text { en } 2001 \text {. }\end{array}$ & $\begin{array}{l}\text { Violación del derecho al } \\
\text { trabajo. } \\
\text { Pérdida de oferta de servi- } \\
\text { cios de salud en Bogotá. } \\
\text { Pérdida de espacios de } \\
\text { práctica para estudiantes } \\
\text { del área de salud. } \\
\text { Proceso de renovación ur- } \\
\text { bana orientado a privatizar } \\
\text { los servicios de salud y a } \\
\text { expulsar a las comunidades } \\
\text { de sus territorios }\end{array}$ \\
\hline
\end{tabular}

Escuela Popular de Salud de la Mesa Intersectorial de Antioquia por el Derecho a la Salud (MIAS)

23 organizaciones de Luchas sindicales, de La MIAS nace a Emergencia social dictada

Trabajadores sindicalizados de la salud, asociación de usuarios de la salud, asociación de pacientes de enfermedades de alto costo, trabajadores informales y movimiento cristiano.

$\begin{array}{lllll}\text { Luchas sindicales, de } & \text { La MIAS nace a } & \text { Emergencia social dictada } & \text { Goce efectivo del dere- } & \text { Local en Mede- } \\ \text { comités de participa- } & \text { partir del } 2009 \text { y } & \text { por el gobierno que restrin- } & \text { cho a la salud para todos. } & \text { Ilín, en articula- } \\ \text { ción en salud, del movi- } & \text { hasta } 2016 \text { había } & \text { gía la autonomía médica e } & \text { Cierre de las brechas } & \text { ción con proce- } \\ \text { miento por la salud. } & \begin{array}{l}\text { realizado tres ver- } \\ \text { incrementaba el gasto de }\end{array} & \text { socio-sanitarias. } & \text { sos nacionales } \\ & \begin{array}{l}\text { sones de escuela } \\ \text { popular en salud. }\end{array} & \text { bolsillo en salud. } & \text { Mejoramiento de los de- } & \text { de lucha por la } \\ & & \text { terminantes de la salud. } & \text { salud. }\end{array}$

Prioridades de las

demandas en salud

Ámbito de

acción

Apertura del HSJD.

Respeto del derecho al

trabajo.

Goce efectivo de derecho

a la salud.

Conservación de la ins-

titución como entidad

pública.

Reconocimiento del

hospital como una ins-

titución insigne de la

enseñanza y la investiga-

ción en salud.

\author{
Local en Bogotá. \\ Se articula con \\ el movimiento \\ estudiantil y \\ movimiento \\ nacional por la \\ salud en algunas \\ coyunturas.
}

\section{Movimiento LGTB}

Organizaciones de per- Luchas por el reconocisonas con orientacio- miento del movimiento nes sexuales no hetero- LGTB. normativas, ONG.
A finales de la

década de 1980

vinculado a los

primeros casos de Sida/ VIH en el país.

\author{
Crímenes de odio como un \\ problema de salud pública. \\ No reconocimiento y dis- \\ criminación en los servicios \\ de salud. \\ Barreras de atención. \\ No inclusión en los planes \\ de beneficio de las necesi- \\ dades específicas de este \\ grupo social. \\ No existencia de modelos \\ de atención diferencial. \\ No valoración de la vida de \\ esta población. \\ Eliminación del estigma y Local en Cali, \\ las barreras de acceso a con articulación \\ servicios de salud. \\ nacional al movi- \\ Reconocimiento de igual miento LGTB, no \\ valor a la vida de este al movimiento de \\ grupo poblacional. salud. \\ Acceso a servicios de \\ salud son barreras.
}

\section{Asociación de trabajadores y ex trabajadores enfermos de Colmotores (ASOTRECOL)}

Trabajadores y extraba- Luchas sindicales. jadores con enfermedades laborales.
2011, posterior al No reconocimiento de la despido de trabaja- enfermedad laboral. dores con enferme- Despido de la empresa. dad laboral.
Falta de valoración de los sindicatos frente a estos problemas de salud

\author{
Reconocimiento y trata- Local en Bogotá. \\ miento de las enferme- \\ dades laborales. \\ Reconocimiento de las \\ prestaciones asistencia- \\ les y económicas.
}




\begin{tabular}{|c|c|c|c|c|c|}
\hline \multicolumn{6}{|l|}{ Cuadro 1. (cont.) } \\
\hline $\begin{array}{l}\text { Tipo de actores que lo } \\
\text { conforman }\end{array}$ & $\begin{array}{l}\text { Tradiciones de lucha } \\
\text { que recoge }\end{array}$ & $\begin{array}{l}\text { Periodo de origen } \\
\text { del proceso }\end{array}$ & $\begin{array}{l}\text { Principales razones para } \\
\text { luchar por la salud }\end{array}$ & $\begin{array}{l}\text { Prioridades de las } \\
\text { demandas en salud }\end{array}$ & $\begin{array}{l}\text { Ámbito de } \\
\text { acción }\end{array}$ \\
\hline \multicolumn{6}{|c|}{ Asociación Campesina del Valle del Rio Cimitarra } \\
\hline $\begin{array}{l}120 \text { Juntas de Acción } \\
\text { Comunal veredales de } \\
\text { municipios del Mag- } \\
\text { dalena Medio Colom- } \\
\text { biano. }\end{array}$ & $\begin{array}{l}\text { Luchas campesinas con } \\
\text { apoyo del movimiento } \\
\text { obrero. }\end{array}$ & $\begin{array}{l}\text { Desde finales de } \\
\text { la década de 1990, } \\
\text { especialmente } \\
\text { vinculada a lucha } \\
\text { por la paz y el } \\
\text { territorio. }\end{array}$ & $\begin{array}{l}\text { Confinamiento de comu- } \\
\text { nidades por el conflicto } \\
\text { armado interno que restrin- } \\
\text { gió acceso a alimentos y } \\
\text { servicios de salud. } \\
\text { Barreras de acceso a servi- } \\
\text { cios de salud y violación del } \\
\text { derecho a la salud. } \\
\text { Defensa del derecho a la } \\
\text { vida. } \\
\text { Derecho a la tierra, al te- } \\
\text { rritorio, al ambiente y a la } \\
\text { soberanía alimentaria }\end{array}$ & $\begin{array}{l}\text { Acceso a la tierra. } \\
\text { Reconocimiento del terri- } \\
\text { torio y la territorialidad. } \\
\text { Garantía para el ejercicio } \\
\text { de los derechos políticos. } \\
\text { Autonomía alimentaria, } \\
\text { reconocimiento de sa- } \\
\text { beres populares, acceso } \\
\text { efectivo a servicios de } \\
\text { salud. }\end{array}$ & $\begin{array}{l}\text { Región del Mag- } \\
\text { dalena Medio } \\
\text { colombiano, } \\
\text { articulado a pro- } \\
\text { cesos nacionales } \\
\text { del movimiento } \\
\text { campesino como } \\
\text { la Cumbre Agra- } \\
\text { ria, las asociacio- } \\
\text { nes de zonas de } \\
\text { reserva campesi- } \\
\text { na; no articulado } \\
\text { a luchas naciona- } \\
\text { les por la salud. }\end{array}$ \\
\hline \multicolumn{6}{|c|}{ Asociación de Cabildos Indígenas del Norte del Cauca (ACIN) } \\
\hline $\begin{array}{l}\text { Cabildos indígenas del } \\
\text { norte del Cauca. }\end{array}$ & $\begin{array}{l}\text { Luchas indígenas desde } \\
\text { la década de } 1960 .\end{array}$ & $\begin{array}{l}\text { Aparece desde la } \\
\text { década de } 1960 \text { y } \\
\text { el tema de salud } \\
\text { se convierte en } \\
\text { central desde } \\
\text { principios de la } \\
\text { década de } 1990 \\
\text { con la reforma del } \\
\text { sistema de salud. }\end{array}$ & $\begin{array}{l}\text { Barreras en el acceso a los } \\
\text { servicios de salud. } \\
\text { No reconocimiento de la } \\
\text { gratuidad en la atención } \\
\text { en salud para los pueblos } \\
\text { indígenas. } \\
\text { No reconocimiento de } \\
\text { saberes ancestrales. } \\
\text { Estigma y maltrato. } \\
\text { Violaciones del derecho a } \\
\text { la salud. }\end{array}$ & $\begin{array}{l}\text { Derecho al territorio. } \\
\text { Autonomía de los pue- } \\
\text { blos. } \\
\text { Desmercantilización de } \\
\text { la salud. } \\
\text { Reconocimiento de un } \\
\text { sistema de salud propio e } \\
\text { intercultural (SISPI). } \\
\text { Valoración de los saberes } \\
\text { ancestrales. } \\
\text { Fortalecimiento de la } \\
\text { identidad propia. }\end{array}$ & $\begin{array}{l}\text { Región del Cau- } \\
\text { ca, articulado } \\
\text { nacionalmente } \\
\text { al movimiento } \\
\text { indígena. Poca } \\
\text { articulación al } \\
\text { movimiento } \\
\text { nacional por la } \\
\text { salud. }\end{array}$ \\
\hline
\end{tabular}

\section{Campañas y abogacía}

Con relación a esta categoría, los estudios de caso mostraron que las campañas se desarrollan en relación con los motivos que detonan la movilización social para cada actor específico. En general todas las campañas reivindican derechos fundamentales, tanto de primera como de segunda generación, y se encuentran necesidades transversales para la movilización como el derecho a la salud, el derecho al trabajo, el reconocimiento de igual valor para todos y todas, el respeto a la dignidad humana, y el acceso oportuno, adecuado, continuo y sin barreras a los servicios de salud y a otros servicios sociales. Estos elementos configuran un núcleo potencial para construir una identidad colectiva nacional en torno al derecho a la salud.

Paralelamente, los casos también ejemplifican las especificidades de las campañas adelantadas por los actores de acuerdo a necesidades propias en salud tales como derecho al reconocimiento de enfermedades laborales, acceso a servicios de atención adecuados para personas con orientaciones sexuales no heteronormativas, reconocimiento y valoración de saberes ancestrales en salud de pueblos indígenas y campesinos, no estigmatización ni maltrato en los servicios de salud. Un último elemento novedoso de las campañas es que algunos actores amplían cualitativamente el significado del derecho a la salud al involucrar 
demandas como el derecho a la tierra, al territorio, a la soberanía alimentaria, al gobierno propio, al buen vivir, al reconocimiento de diferentes epistemologías y a asignar igual valor a todos los cuerpos.

Frente a la abogacía, se encontró que los actores a pesar de la visibilidad de las campañas, aún no logran una fuerte incidencia en la política pública. Los logros más importantes los concentra el movimiento indígena, que cuenta con una Mesa Permanente de Negociación con el gobierno nacional y ha logrado el reconocimiento de un sistema propio e intercultural en salud; el movimiento LGTB, que logró el diseño de la primera política pública departamental, que incluyó la salud en la agenda; la aprobación de una zona de reserva campesina en el Valle del Río Cimitarra en el marco de la defensa de la vida, el territorio y la paz; y la lucha nacional que logró la derogatoria de algunas normas nacionales en 2010 y el reconocimiento del derecho a la salud como fundamental en 2015. En el ámbito internacional, solo la asociación de trabajadores y ex trabajadores enfermos de la General Motors, aprovechando la negociación del Tratado de Libre Comercio, logró visibilizar su problemática con apoyo de sindicatos de Estados Unidos, obteniendo solidaridad económica ${ }^{23}$.

\section{La generación, diseminación y uso de conocimiento}

Se pueden resaltar algunos elementos comunes en los casos estudiados con relación a esta categoría. En el primer asunto -generación de conocimiento- ha sido especialmente importante la recuperación y fortalecimiento de saberes propios -de pueblos indígenas y campesinos- para contribuir a la salud para todos.Así mismo la constitución de redes organizativas gremiales con instituciones locales y académicas que, a través de investigación acción participativa, permitieron que procesos como los del Hospital San Juan de Dios, el movimiento indígena del Norte del Cauca, el movimiento campesino, la Escuela Popular por la Salud y el mismo movimiento por la salud, cuenten con investigaciones de académicos, estudiantes y activistas que cualifican los argumentos para la contienda por el derecho a la salud y otros derechos (cuadro 2).

Frente al segundo asunto -diseminación del conocimiento- los casos muestran cuatro grandes tipos de estrategias: i) procesos de educación popular guiados por propuestas como las de Paulo Freire que buscan fortalecer la apropiación de conocimientos adquiridos y recuperados por los actores; ii) el uso de medios alternativos de comunicación como programas radiales, páginas web, noticieros, etc.; iii) el uso innovador de estrategias artísticas como obras de teatro y canciones, entre otras; y iv) estrategias de formación cara a cara, transversal para todos los actores. Finalmente, el conocimiento ha sido usado especialmente para cualificar los argumentos políticos frente a adversarios como el gobierno nacional o local, empresas de salud -tanto aseguradoras como prestadoras de servicios sanitarios- y para fortalecer los liderazgos que emergen en la búsqueda de salud para todos. 
Cuadro 2. Formas de producción, diseminación y uso de conocimiento

\begin{tabular}{ll}
\hline Caso & Conocimiento generado \\
\hline Movimiento Nacional por & Reconocimiento de prácticas de resis- \\
la Salud y la Seguridad & tencia de actores subalternos. \\
Social & Construcción de ciudadanía en salud. \\
& Análisis del proceso de reforma y del \\
& sistema de salud. Análisis de la exigibi- \\
& lidad del derecho a la salud. \\
& Situación del sector salud. \\
Hospital San Juan de Dios & Estudios jurídicos e históricos sobre \\
(HSJD) & el HSJD.
\end{tabular}

\section{Estrategias de diseminación}

Cartillas educativas dirigidas a organizaciones y movimientos sociales. Escuelas populares en salud. Artículos académicos.

Conversatorios. Recorridos por el hospital. Exposiciones artísticas. Boletines. Galerías.

Escuela Popular de Salud de la Mesa Intersectorial de Antioquia por el Derecho a la Salud (MIAS)

Movimiento LGTB
Ciudadanía en salud. Identificar el trabajo de grupos subalternos. Proveer conocimiento asequible a las propias organizaciones.

Sobre violaciones del derecho a la vida y otros derechos humanos.
Asociación de trabajadores y ex trabajadores enfermos de Colmotores (ASOTRECOL)

Asociación Campesina del Valle del Rio Cimitarra
Asociación de Cabildos Indígenas del Norte del Cauca (ACIN)
Conocimiento médico- legal sobre las patologías que afectan a los obreros.

Recuperación de fuentes de agua contaminadas con mercurio producto de la minería. Proyectos de energías alternativas a bajo costo. La caracterización biológica de la Línea Amarilla (línea trazada por la comunidad para delimitar zonas de protección ambiental). Caracterización de la Ciénaga de San Lorenzo.

Recuperación de saberes ancestrales en salud.

Recuperación de la cosmovisión propia.

Recuperación de prácticas rituales.

Análisis de condiciones de salud y del territorio.
Plantones por la salud. Procesos de educación popular. Programas radiales. Obras de teatro.

Observatorio de violaciones de Derechos Humanos y el Observatorio de violación del derecho a la salud, con especial énfasis en la vulneración de este derecho a las mujeres trans.

Asesorías a los trabajadores para confrontar fallos judiciales.

Página web y redes sociales.

Escuelas de liderazgo. Página web. Marcha TV. Agencia Prensa Rural. Comunidades campesinas en resistencia

Procesos de capacitación comunitaria. Medios alternativos como emisora radial y página web.
Uso del conocimiento

Para la contienda en el campo de la salud.

Formación de actores sociales.

Defensa de los derechos de los trabajadores.

Reconocimiento del carácter público de la institución.

La exigencia de su reapertura.

Concientizar nuevos actores. Denunciar violaciones de derechos humanos y del derecho a la salud. La contienda por la salud.

Denunciar violaciones de derechos humanos en su población.

Negociar políticas públicas de reconocimiento.

Exigibilidad jurídica del derecho a la salud en el trabajo frente a actores como Ministerio de Trabajo.

Denuncia nacional e internacional.

Protección del medio ambiente. Análisis de la situación en sus territorios. Diseño de planes de vida de la asociación. Exigibilidad social y jurídica de los derechos. Negociación frente al Estado.

Denunciar la situación de salud de las comunidades.

Exigibilidad social y jurídica del derecho a la salud.

Construcción de programas de salud adecuados interculturalmente. Procesos de negociación con gobiernos regionales o nacionales. Diseño de Planes de Vida. Fortalecimiento de la identidad.
Hasta aquí podemos afirmar que la genera-
ción de conocimiento aportó a identificar no
sólo los efectos de la mercantilización de los
servicios de salud, sino también del modelo
de desarrollo y su relación con la salud de las poblaciones; y a visibilizar las luchas y formas de resistencia de actores subalternos, las maneras de construir ciudadanía en salud y los aportes colectivos a la salud para todos. Así mismo, demostró que existen algunos 
vínculos entre academia, organizaciones de la sociedad civil y movimientos sociales por la cualificacióny la construcción de argumentos para la defensa del derecho a la salud y de otros derechos. Además, los actores han intentado innovar en las formas de diseminación del conocimiento, utilizando estrategias educativas, artísticas y tecnológicas para ello.

\section{Entrenamiento y construcción de capacidades}

El entrenamiento y la construcción de capacidades se ha desarrollado en los casos analizados a partir de cuatro grupos de estrategias: primera, el acceso de líderes de todos los procesos sociales a educación formal, tanto a nivel de secundaria como de educación superior; así, en todos ellos se encuentran profesionales propios formados y vinculados a procesos organizativos, producción de conocimiento y planeación local. Segunda, el desarrollo de experiencias de educación popular, las cuales han transitado en temáticas como derechos humanos, derecho a la salud, mecanismos de exigibilidad jurídica de derechos, uso de medios de comunicación alternativos, generación de conocimiento, protección del territorio, género y diversidad sexual, entre otras consideradas pertinentes. Tercera, la formación de recurso propio en salud, promotoras y promotores de salud, parteras y agentes de salud indígenas, quienes aportan a partir del diálogo de saberes y la interculturalidad a la salud de sus comunidades. Cuarta, y esto es quizás lo que más se debe resaltar entre el movimiento indígena y campesino, la formación de capacidades en los propios procesos de organización y lucha: algunos de los líderes se empiezan a formar en la juventud participando de los diferentes comités y espacios organizativos, aprendiendo de las experiencias de quienes les anteceden desde la práctica y la reflexión sobre la misma. Muchos procesos de construcción de capacidades han contado con el apoyo de organizaciones no gubernamentales o académicas, ratificando los vínculos encontrados entre estos y las organizaciones de la sociedad civil.

Finalmente, es importante reconocer que la formación de capacidades para la exigibilidad del derecho a la salud, al territorio, a la soberanía alimentaria, a la vida y a cosmovisiones y prácticas propias, ha estado mediada por los contextos locales -políticos, económicos y culturales-, y ha incluido una gran diversidad de expresiones construidas por los diferentes actores sociales, en un contexto de desigualdad, violencia, persecución y resistencia.

\section{Diálogo sobre las políticas y el invo- lucramiento de la sociedad civil en la gobernanza en salud}

Esta categoría mostró que se ha venido construyendo y consolidando una noción compleja de derecho fundamental a la salud y de salud para todos, que no existía antes de los procesos de reforma sectorial, y que involucra no sólo el componente prestacional sino la garantía de otros derechos fundamentales para el buen vivir; esta construcción ha permitido la cualificación de la contienda nacional por parte de los actores subalternos de la sociedad civil y la incidencia en algunos momentos en la política pública.

Los casos más importantes de esta incidencia en las políticas públicas nacionales están representados en la derogatoria de la Emergencia Social en 2010, durante el gobierno de Álvaro Uribe Vélez, decretada para profundizar la mercantilización de la salud; la promulgación de la Ley Estatutaria de Salud en el año 2015 pese a la clara oposición del gobierno nacional; el reconocimiento del Sistema Indígena de Salud Propio e Intercultural para los pueblos indígenas; y los múltiples llamados de la Corte Constitucional y la Defensoría del Pueblo ante la violación sistemática del derecho a la salud en Colombia. Por el contrario, el proceso de estas organizaciones aún no se ha enfocado en la gobernanza global de la salud.

En síntesis, si bien las organizaciones y movimientos sociales de la sociedad civil 
colombiana no han logrado reversar completamente las reformas de mercado en salud, sí han logrado evitar la profundización de las mismas, han ampliado el significado y contenido del derecho a la salud y de la salud para todos presionando al Estado en este sentido, han ayudado a defender el derecho al territorio, a la vida y a la paz, y han avanzado en la construcción de una ciudadanía sanitaria aún incipiente.

\section{Conclusiones}

Los casos de Colombia estudiados muestran un enriquecido y heterogéneo panorama de involucramiento de procesos organizativos y movimientos sociales de la sociedad civil en la lucha por la salud para todos y todas en el ámbito local, regional y nacional, como ha sido documentado en investigaciones previas ${ }^{10,11}$.

Este involucramiento, en búsqueda de soluciones acuciantes a diversas necesidades de salud, cruza distintas temáticas relacionadas con ésta, determina modos diversos de acción colectiva según las condiciones de clase, género, étnicas, laborales y ciudadanas de los participantes, contextos y actores disímiles constituidos por las políticas de mercantilización del derecho a la salud, la exclusión social y la violencia sociopolítica, lo que también ha ocurrido en otros contextos de América Latina9.

Igualmente, muestran variadas formas autóctonas de involucramiento de la sociedad civil, elementos de práctica (estructuras, recursos y estrategias), resistencias y logros, a pesar de que no hayan sido específicamente promovidas por el Movimiento Nacional por la Salud y la Seguridad Social ni por el Movimiento de la Salud de los Pueblos, o no se hayan inscrito en sus programas de lucha.

Es por ello que, por ejemplo, componentes como la formación de capacidades, la generación y difusión de conocimientos, el diálogo para las políticas y la gobernanza, el desarrollo de campañas y la construcción de dinámicas organizativas y movimiento, han incluido gran diversidad de formas construidas por los propios actores sociales según las circunstancias y contextos de desigualdad social, discriminación, persecución, violencia sociopolítica y resistencia en que les ha correspondido trabajar y vivir. De esta manera, en los casos que hemos examinado, cada proceso ha respondido a necesidades particulares para el despliegue de la acción colectiva que incluyen la defensa de la vida, los territorios, la soberanía alimentaria, las identidades y autonomías, el trabajo, la protección de la salud en el trabajo, la defensa de las instituciones públicas de salud, la atención en salud y el reconocimiento a la diferencia, enriqueciendo la perspectiva frente a los campos de lucha por la salud ${ }^{5}$.

La forma y contenido de cada uno de los componentes relacionados con el involucramiento de la sociedad civil en el logro de la salud para todos y todas en los escenarios urbanos ha sido diversa y ha estado relacionada con procesos sociales identificados con: la lucha por el derecho a la salud en el trabajo; la resistencia a procesos de privatización y mercantilización de la atención en salud y a dinámicas de despojo determinadas por proyectos de renovación urbana ligados a estrategias de generación de negocios de atención en salud y de turismo médico; el reconocimiento del derecho a la salud en el marco dela reivindicación de la ciudadanía social de las diversidades sexuales; y, más generalmente, la disputa por la orientación y el contenido del sistema de salud y seguridad social, sus garantías prestacionales y de acceso a la atención sanitaria.

En los escenarios rurales, agrarios y mineros, la forma y contenido de las luchas y la forma de involucramientos por el logro de la salud de organizaciones y movimientos sociales ha estado más vinculada a las relaciones tejidas entre éstos y las fuerzas y organizaciones políticas que han orientado sus dinámicas de lucha campesina e indígena para garantizarse formas populares y propias de atención en 
salud. Ello ante la ausencia de servicios en salud oficiales o el reclamo de autonomía de los pueblos indígenas y campesinos, la creación de condiciones propias, sociales, ambientales y culturales para la salud y la soberanía alimentaria y seguridad nutricional.

En este estudio también encontramos que los procesos sociales locales no muestran vínculos directos entre sí, ni usualmente con los movimientos nacionales específicos que han emergido por el derecho a la salud y a la seguridad social en Colombia, como tampoco lo tienen con las dinámicas latinoamericanas y globales explícitas del Movimiento de Salud de los Pueblos. No obstante lo cual, han estado influenciados de algún modo tanto por las luchas del Movimiento Nacional por la Salud y la Seguridad Social como por las orientaciones y enfoques del Movimiento de Salud de los Pueblos, a través de activistas nacionales y locales, por ejemplo estudiantes, vinculados con ellos, especialmente en los espacios urbanos, campesinos y étnico-indígenas.

Explorar los temas sobre construcción de capacidades, generación y difusión de conocimiento, construcción de movimiento, entre otros, en estos procesos locales, también contribuye a comprender los modos particulares cómo la sociedad civil se vincula al logro de la salud para todos aún en circunstancias de alta represión y violencia contra fuerzas que disputan hegemonías de clase, étnicas y de género.

Finalmente, el conjunto de experiencias que aporta el caso colombiano muestra de manera clara que el logro de salud para todos y del derecho a la salud no se restringen únicamente al componente prestacional de servicios asistenciales, sino que resulta más amplio al incorporar la garantía de derechos individuales y colectivos como el derecho al territorio, a la seguridad alimentaria, al trabajo, al reconocimiento y respeto a la diferencia y a la participación plena y decisiva de actores sociales subalternizados.

\section{Agradecimientos}

Agradecemos a todas las personas que estuvieron involucradas en el desarrollo de esta investigación: Katherine Cuéllar Bravo, Jenny Cristina Gutiérrez García, Deisy Paola Carranza López, María Alejandra Contreras Sánchez, Diana Yadira Almonacid Rojas, KatherinIovanowa Carrillo Noguera, Juan Pablo López M, Gloria Amparo Yonda, Luz Ángela Palacios y Alexandra de la Cruz. También agradecemos al conjunto de organizaciones sociales que abrieron sus espacios para poder investigar sobre sus procesos colectivos. Asimismo, agradecemos al Movimiento de Salud de los Pueblos que contribuyó con los recursos del proyecto 'La contribución de la sociedad civil al logro de la salud para todos' para realizar esta investigación.

\section{Colaboradores}

Torres-Tovar M (0000-0002-6232-6706)* contribuyó substancialmente en la concepción, planificación y análisis del manuscrito; significativamente en la elaboración del borrador y en la revisión crítica del contenido; y en la aprobación de su versión final. Vega-Romero RR (0000-0002-7632-6723)* contribuyó substancialmente en la concepción, planificación y análisis del manuscrito; significativamente en la elaboración del borrador y en la revisión crítica del contenido; y en la aprobación de su versión final. Luna-García JE (0000-00020885-7223)* contribuyó con la concepción y planificación, elaboración del contenido y sus correcciones, así como en la aprobación de su versión final. Borrero-Ramírez YE (00000003-2559-4637)* contribuyó al diseño de la investigación, trabajo de campo, análisis y discusión de la información de casos, escritura y revisión crítica del manuscritoy EcheverryLópez ME (0000-0001-9116-5107)* contribuyó a la elaboración de borrador del manuscrito y a la revisión crítica de su contenido. 


\section{Referencias}

1. Giovanella L, Almeida PF, Vega Romero R, et al. Panorama de la Atención Primaria de Salud en Suramérica: concepciones, componentes y desafíos. Saúde debate [internet]. 2015 [acceso en 2019 Mayo 15];39(105):300322. Disponible en:http://www.scielo.br/pdf/sdeb/ v39n105/0103-1104-sdeb-39-105-00300.pdf.

2. Hone T, Macinko J, Millett C. Revisiting Alma-Ata: what is the role of primary health care in achieving the Sustainable Development Goals? The Lancet [internet]. 2018 [acces oen 2019 Mayo 15]; 392(10156):14611472. Disponible en: https://doi.org/10.1016/S01406736(18)31829-4.

3. Meisterhans N. Salud para todos: implementación del derecho a la salud en la agenda post-2015. Perspectivas desde el hemisferio Sur. Medicina Social [internet]. 2014 [acceso en 2019 Mayo 18];9(3):213-231. Disponible en: http://www.socialmedicine.info/index.php/medicinasocial/article/viewFile/868/1636.

4. San Sebastián M, Hurtig A-K, Breilh J y Quizhpe A. El Movimiento de Salud de los Pueblos: salud para todos ya. Rev PanamSaludPublica/Pan Am J Public Health [internet]. 2005 [acceso en 2019 feb 16]; 18(1):45-49. Disponible en: http://iris.paho.org/ xmlui/bitstream/handle/123456789/8078/27088. pdf? sequence $=1 \&$ is Allowed $=y$.

5. Sengupta A, Bodini C, Franco S. Struggles for Health: An Emancipatory Approach in the Era of Neoliberal Globalization.Development [internet]. 2018. [acceso en 2019 Mayo 16]. Disponible en: https://doi. org/10.1057/s41301-018-0196-Z.

6. People's Health Movement. Civil Society engagement for health for all. Guideline for country teams for phase 1 of the action -research. [internet]. 2014. [acceso en 2019 abr12]. Disponible en: https://phmovement. org/wp-content/uploads/2018/06/Countries_Stage1GuidelinesForCountryTeams.pdf.

7. Bodini C, Sanders D, Sengupta A. The contribution of civil society engagement to the achievement of Health for All (CSE4HFA). [internet]. 2018 [acceso en 2019 feb 25]. Disponible en: https://phmovement. org/wp-content/uploads/2018/07/CSE4HFA_FinalReport_Long_180909.pdf.

8. Aguilera R. Las transformaciones del Estado contemporáneo: legitimidad del modelo de Estado neoconstitucional. Universitas [internet]. 2012 [acceso en 2019 feb 16]; (15):3-25. Disponible en: http://universitas.idhbc.es/n15/15-02.pdf.

9. Homedes N, Ugalde A. Las reformas de salud neoliberales en América Latina: una visión crítica a través de dos estudios de caso. Rev. panam. salud pública. [internet]. 2005 [acesso en 2019 mar 11];17(3):210220. Disponible en: https://scielosp.org/pdf/rpsp/ v17n3/a12v17n3.pdf.

10. Echeverry-López ME, Borrero-Ramírez YE. Protestas sociales por la salud en Colombia: la lucha por el derecho fundamental a la salud, 1994-2010. Cad. Saúde Pública.[internet]. 2015 [acesso en 2019 mar 14]; 31(2):354-364. Disponibleen: http://www.scielo.br/ pdf/csp/v31n2/0102-311X-csp-31-02-00354.pdf.

11. Torres-Tovar, M. Lucha social contra la privatización de la salud. Bogotá, DC: CINEP; 2013.

12. Borrero-Ramírez YE, Torres-Tovar M, Echeverry-López ME. Salud para Todos. La construcción del movimiento nacional por la salud y la seguridad social, caso Colombia 1990-2015. En: Vega-Romero R, Torres-Tovar M, Luna-García JE, organizadores. Luchas sociales por la salud en Colombia. Bogotá,DC: Universidad Nacional de Colombia; 2019.

13. Carranza López DP, Contreras Sánchez MA, Almonacid Rojas DY. Luchas invisibles: un estudio sobre el proceso de resistencia de los trabajadores del Hospital San Juan de Dios. En: Vega-Romero R, Torres-Tovar M, Luna-García JE, organizadores. Luchas sociales por la salud en Colombia. Bogotá, DC: Universidad Nacional de Colombia; 2019.

14. Molano P, Hoyos SA, Cano M, et al. Construcción colectiva del derecho a la salud: Una iniciativa desde la 
educación popular. Medellín: Universidad de Antioquia; 2016.

15. Cuéllar Bravo K, Borrero-Ramírez Y. Construcción de ciudadanía en salud en el marco del movimiento social LGBT en la ciudad de Cali. En: Vega-Romero $\mathrm{R}$, Torres-Tovar M, Luna-García JE, organizadores. Luchas sociales por la salud en Colombia. Bogotá, DC: Universidad Nacional de Colombia; 2019.

16. Torres-Tovar M, Luna-García JE. Asociación de Trabajadores y Extrabajadores Enfermos de Colmotores-ASOTRECOL: un caso de lucha por el derecho a la salud en el trabajo en Colombia. En: Vega-Romero R, Torres-Tovar M, Luna-García JE, organizadores. Luchas sociales por la salud en Colombia. Bogotá, DC: Universidad Nacional de Colombia; 2019.

17. López JP. La salud y la vida en la comunidad rural del Valle del Rio Cimitarra. Sistematización de la experiencia organizativa en salud por parte de la Asociación Campesina del Valle del Rio Cimitarra (ACVC). 1980-2016. En: Vega-Romero R, Torres-Tovar M, Luna-García JE, organizadores. Luchas sociales por la salud en Colombia. Bogotá, DC: Universidad Nacional de Colombia; 2019.

18. Carrillo Noguera KI. Soberanía alimentaria para las comunidades campesinas del Magdalena Medio en la Zona de Reserva Campesina del Valle del Río Cimitarra. En: Vega-Romero R, Torres-Tovar M, Luna-García JE, organizadores. Luchas sociales por la salud en Colombia. Bogotá, DC: Universidad Nacional de Colombia; 2019.

19. Gutiérrez García JC. Las mujeres campesinas y su contribución al logro de la salud para sus comunidades; el caso de la Asociación Campesina del Valle del río Cimitarra. En: Vega-Romero R, Torres-Tovar M, Luna-García JE, organizadores. Luchas sociales por la salud en Colombia. Bogotá, DC: Universidad Nacional de Colombia; 2019.

20. Yonda GA, Palacios LA, De la Cruz A, et al. Aportes del movimiento indígena del Norte del Cauca a la construcción de salud para todos. Una mirada desde las autoridades tradicionales y los dinamizadores del tejido de salud de la ACIN. En: Vega-Romero R, Torres-Tovar M, Luna-García JE, organizadores. Luchas sociales por la salud en Colombia. Bogotá, DC: Universidad Nacional de Colombia; 2019.

21. Loewenson R, Laurell AC, Hogstedt C, et al. Participatory action research in health systems. A methods reader. Harare: TARSC, AHPSR, WHO, IDRC Canadá, EQUINET [internet]. 2014. [acesso en 2019 mar 14]. Disponible en: http://www.equinetafrica.org/sites/default/files/uploads/documents/PAR\%20Methods\%20Reader2014\%20for\%20web.pdf.

22. Echeverry-López ME, organizadora. Indignación justa: Estudios sobre la Acción de Tutela en Salud en Medellín. Medellín: Facultad Nacional de Salud Pública - Universidad de Antioquia; 2013.

23. Torres-Tovar M, Luna-García JE, Parra J, et al. Acción colectiva por el derecho a la salud en el trabajo: el caso de Asotrecol. En: Gallo O, Castaño E, editores. La salud laboral en el siglo XX y el XXI: De la negación al derecho. Medellín: Escuela Nacional Sindical; 2016.

Recebido el 09/05/2019

Aprovado el 16/09/2019

Conflicto de intereses: inexistente

Apoyo financiero: no hubo 